\title{
Application of the Adomian Decomposition Method to Oscillating Viscous Flows
}

\author{
Chi-Min Liu \\ Division of Mathematics, General Education Center, Chienkuo Technology University, Changhua City, Taiwan
}

Email address:

cmliu@ctu.edu.tw

\section{To cite this article:}

Chi-Min Liu. Application of the Adomian Decomposition Method to Oscillating Viscous Flows. Applied and Computational Mathematics. Vol. 5, No. 3, 2016, pp. 121-132. doi: 10.11648/j.acm.20160503.15

Received: June 3, 2016; Accepted: June 13, 2016; Published: June 29, 2016

\begin{abstract}
In this paper three oscillating viscous flows are studied by applying the Adomian decomposition method (ADM). Major improvement is on the choice of the assignment of the first term of the decomposition series. Different from past studies in which the initial velocity profile of the whole domain is assigned as the first term of the decomposition series, the assignment in present study is simply the boundary velocity for Stokes' second problem and the pressure gradient for pulsatile flows. This improvement demonstrates and implies that ADM is not only good in approaching the known exact solution, but also possesses the practicability in treating realistic problems. The derived approximate solutions accurate up to any order can be obtained after two key parameters are determined. Present results show an excellent agreement with those calculated by the exact solutions. Based on the present results, more periodic problems can be analyzed by ADM with the help of Fourier analysis.
\end{abstract}

Keywords: Adomian Decomposition Method, Stokes' Second Problem, Pulsatile Flow, Starting Assignment

\section{Introduction}

Adomian decomposition method (ADM) [1-2] is a powerful method to analyze mathematical and practical problems. Without the requirements of discretization, linearization and perturbation, ADM can be applied to solve linear or nonlinear, ordinary or partial differential equations and integral equations for either initial-value or boundary-value problems. A great deal of efforts has been devoted to the applications of ADM [3-11], the improvement associated with Padé approximation [12-14], and the examination of the convergent behavior [15-16].

Recently, Liu [17] applied ADM to investigate Stokes' second problem and planar pulsatile flow in a semi-infinite domain. He assigned the first term of the decomposition series from the known exact solutions [18-19] of the velocity and the shear stress at the plate for Stokes' second problem, and the pressure gradient for pulsatile flow. Using the differential operator with respect to the spatial coordinate, the derived solution is displayed in terms of powers in spatial variable. Similarly, Farkhadnia et al. [20] also applied ADM and other two methods to study Stokes' second problem. The starting assignment of the first term of the decomposition series (Eq.(64)) is the velocity profile at $t=0$ which is directly adopted from the exact solution [21-22]. Different from Liu's solution [17], the solution is shown in terms of powers in time due to the different definition of the differential operator they chose. Though Liu and Farkhadnia et al. successfully validated the efficiency and accuracy of ADM by assigning the first term of the decomposition series acquired from the exact solution, however, the ability of ADM for simulating realistic flows by simply assigning the given boundary velocity (for Stokes' problem) or the pressure gradient (for pulsatile flow) as the first term of the series is not well examined.

Due to aforementioned demonstration, Stokes' second problem in a finite-depth domain, pulsatile flows between plates and in a circular tube will be studied by applying ADM. Results for three cases are respectively shown in Sections 2 to 4. Each Section includes the derivation processes of the ADM solution and the examination of convergent behavior of two key parameters. The derived solution will be compared with the exact solution to verify the efficiency and accuracy of ADM. Conclusions are made in Section 5. 


\section{Stokes' Second Problem in A Finite-Depth Domain}

Stokes' problem [23], also known as Rayleigh problem [24], describes that a viscous fluid which occupies a semi-infinite domain is driven by a moving plate below. The plate moving in a constant speed or in an oscillation type is named as the first or second problem. Analytical solutions to the flow velocity [18-19, 25] and the wall stress [19] were well investigated and discussed. Extended from the semi-infinite domain of fluid, Stokes' problem for a fluid in a finite-depth domain was studied [26]. The flow considered is bounded by a free surface above and a moving plate below. Solutions of the first and second problems are derived and shown in the form of infinite series. For the second problem, the exact solution can be separated into the steady-state and the transient parts. In addition to analytical investigation, numerical methods based on discretization schemes, which include finite-difference method [27], finite-element method [28] and mesh-free numerical scheme [29], are performed to analyze the semi-infinite Stokes' problems. In general numerical results show a good agreement with analytical solutions.

In this section, ADM is applied to solve Stokes' second problem in a finite-depth domain by simply assigning the plate velocity to the first term of the decomposition series instead of those adopted in [17] and [20]. Consider a fluid of kinematic viscosity $\boldsymbol{v}$ which is bounded by a plate located at $y=0$ and a free surface at $y=h$. The fluid is driven by the oscillating plate with the speed $u_{0} \cos \omega t$. The momentum equation and boundary conditions for the velocity $u$ are

$$
\begin{gathered}
\frac{\partial u}{\partial t}=v \frac{\partial^{2} u}{\partial y^{2}} \\
u(y=0)=u_{0} \cos \omega t \\
\frac{\partial u}{\partial y}(y=h)=0
\end{gathered}
$$

Dimensional parameters

$$
U=\frac{u}{u_{0}}, Y=\frac{y}{h}, T=\omega t, \lambda=\frac{v}{\omega h^{2}},
$$

are defined and applied to Eqs.(1) to (3). The results are

$$
\begin{aligned}
& \frac{\partial U}{\partial T}=\lambda \frac{\partial^{2} U}{\partial Y^{2}}, \\
& U(Y=0)=\cos T . \\
& \frac{\partial U}{\partial Y}(Y=1)=0 .
\end{aligned}
$$

The value of $\lambda$ will be assigned to be unity henceforward without loss of generality. To apply ADM, a differential operator

$$
\mathrm{L}_{Y}(\cdot) \equiv \frac{\partial^{2}}{\partial Y^{2}}(\cdot)
$$

is defined and then Eq.(5) can be shown as

$$
\mathrm{L}_{Y} U=\frac{\partial U}{\partial T} \text {. }
$$

Applying the inverse operation of Eq.(8)

$$
\mathrm{L}_{Y}^{-1}(\cdot)=\int_{0}^{Y} \int_{0}^{Y}(\cdot) \mathrm{d} Y \mathrm{~d} Y .
$$

to Eq.(9) results in

$$
U(Y, T)=U(0, T)+Y \cdot U_{Y}(0, T)+\int_{0}^{Y} \int_{0}^{Y} \frac{\partial U}{\partial T} \mathrm{~d} Y \mathrm{~d} Y
$$

The solution of $U$ is assumed to be

$$
U(Y, T)=\sum_{n=0}^{\infty} U_{n}(Y, T)
$$

By using the boundary condition Eq.(6), the first term $U_{0}$ is assigned as

$$
U_{0}=\cos T+Y \cdot F(T)
$$

where $F(T)$ represents the velocity gradient at $Y=0$ which is an unknown function of $T$ and will be determined later. The remaining terms at the right-hand side of Eq.(12) can be recursively calculated by

$$
U_{n+1}=\int_{0}^{Y} \int_{0}^{Y} \frac{\partial U_{n}}{\partial T} \mathrm{~d} Y \mathrm{~d} Y . n \geq 0 .
$$

Now $U_{1}$ to $U_{4}$ are shown below for clear comprehension

$$
\left\{\begin{array}{l}
\boldsymbol{U}_{1}=-\frac{\boldsymbol{Y}^{2}}{2 !} \sin \boldsymbol{T}+\frac{\boldsymbol{Y}^{3}}{3 !} \boldsymbol{F}^{\prime}(\boldsymbol{T}) \\
\boldsymbol{U}_{2}=-\frac{\boldsymbol{Y}^{2}}{4 !} \cos \boldsymbol{T}+\frac{\boldsymbol{Y}^{5}}{5 !} \boldsymbol{F}^{\prime \prime}(\boldsymbol{T}) \\
\boldsymbol{U}_{3}=+\frac{\boldsymbol{Y}^{6}}{6 !} \sin \boldsymbol{T}+\frac{\boldsymbol{Y}^{7}}{7 !} \boldsymbol{F}^{\prime \prime \prime}(\boldsymbol{T}) \\
\boldsymbol{U}_{4}=+\frac{\boldsymbol{Y}^{8}}{8 !} \cos \boldsymbol{T}+\frac{\boldsymbol{Y}^{9}}{9 !} \boldsymbol{F}^{(4)}(\boldsymbol{T})
\end{array}\right.
$$


where the superscript $(i)$ demotes the $i$-th differentiation

complete solution can be written as

with respect to $T$, and ! the factorial. Therefore the

$$
U(Y, T)=\sum_{i=0}^{\infty} \frac{Y^{2 i+1} F^{(i)}(T)}{(2 i+1) !}+\cos T \cdot \sum_{i=0}^{\infty} \frac{(-1)^{i} Y^{4 i}}{(4 i) !}+\sin T \cdot \sum_{i=0}^{\infty} \frac{(-1)^{i+1} Y^{4 i+2}}{(4 i+2) !} .
$$

Inserting Eq.(16) into Eq.(7) gives

$$
\sum_{i=0}^{\infty} \frac{F^{(i)}(T)}{(2 i) !}+\cos T \cdot \sum_{i=0}^{\infty} \frac{(-1)^{i+1}}{(4 i+3) !}+\sin T \cdot \sum_{i=0}^{\infty} \frac{(-1)^{i+1}}{(4 i+2) !}=0
$$

As the plate oscillates steadily, $F(T)$ is assumed to be

$$
F(T)=a \cos T+b \sin T,
$$

where $a$ and $b$ are constant parameters to be determined. Substituting Eq.(18) into Eq.(17) results in

$$
\begin{aligned}
& \left\{a \cdot \sum_{i=0}^{\infty} \frac{(-1)^{i}}{(4 i) !}+b \cdot \sum_{i=0}^{\infty} \frac{(-1)^{i}}{(4 i+2) !}+\sum_{i=0}^{\infty} \frac{(-1)^{i+1}}{(4 i+3) !}\right\} \cdot \cos T \\
& +\left\{a \cdot \sum_{i=0}^{\infty} \frac{(-1)^{i+1}}{(4 i+2) !}+b \cdot \sum_{i=0}^{\infty} \frac{(-1)^{i}}{(4 i) !}+\sum_{i=0}^{\infty} \frac{(-1)^{i+1}}{(4 i+1) !}\right\} \cdot \sin T=0
\end{aligned}
$$

Consider the approximate solution collecting terms up to $U_{n}$, we define

$$
\Phi_{n}=\sum_{i=0}^{n} U_{n}(Y, T)
$$

Now parameters $a$ and $b$ for $\Phi_{n}$ can be solved by

$$
\left\{\begin{array}{l}
a \cdot \sum_{i=0}^{i \leq \frac{n}{2}} \frac{(-1)^{i}}{(4 i) !}+b \cdot \sum_{i=0}^{i \leq \frac{n}{2}-\frac{1}{2}} \frac{(-1)^{i}}{(4 i+2) !}=\sum_{i=0}^{i \leq \frac{n}{2}-\frac{3}{4}} \frac{(-1)^{i}}{(4 i+3) !} \\
a \cdot \sum_{i=0}^{i \leq \frac{n}{2}-\frac{1}{2}} \frac{(-1)^{i+1}}{(4 i+2) !}+b \cdot \sum_{i=0}^{i \leq \frac{n}{2}} \frac{(-1)^{i}}{(4 i) !}=\sum_{i=0}^{i \leq \frac{n}{2}-\frac{1}{4}} \frac{(-1)^{i}}{(4 i+1) !}
\end{array}\right.
$$

For example, if $\Phi_{2}$ is considered, parameters $a$ and $b$ can be determined by

$$
\left\{\begin{array}{l}
a \cdot\left(1-\frac{1}{4 !}\right)+b \cdot\left(\frac{1}{2 !}\right)=\frac{1}{3 !} \\
a \cdot\left(-\frac{1}{2 !}\right)+b \cdot\left(1-\frac{1}{4 !}\right)=1
\end{array}\right.
$$

and the corresponding solutions are

$$
\left\{\begin{array}{l}
a=-\frac{196}{673} \cong-0.2912 \\
b=\frac{600}{673} \cong 0.8915
\end{array}\right.
$$

Based on above derivation, the approximate solution Eq.(20) accurate up to any order can be solved after parameters $a$ and $b$ are determined by Eq.(21).

Based on the derived solution, the convergent behavior of parameters $a$ and $b$ is firstly examined. The solution pairs of $(a, b)$ for $\Phi_{1}$ to $\Phi_{6}$ by solving Eq.(21) are listed in Table 1. In each column, the decimal is the approximate value 
and the fraction in the bracket is the exact value solved from Eq.(21). It is clearly found that solutions of $a$ and $b$ will converge when $n$ becomes large.

$$
U_{\text {exact }}=1+\cos T+2 \cdot \sum_{n=0}^{\infty}\left[\frac{\sin N Y}{N} \cdot \frac{N^{2} \sin T-\cos T}{1+N^{4}}\right], N=\frac{2 n+1}{2} \pi,
$$

are plotted in Figure 1 for comparison. Velocity distributions along $Y$ at $T=0, \pi / 6, \pi / 3$ and $\pi / 2$ are respectively displayed while the period of oscillation is $2 \pi$. $\Phi_{1}, \Phi_{2}$ and $\Phi_{4}$ are respectively drawn in green, blue and red solid lines and $U_{\text {exact }}$ is displayed in black dash line. It shows that $\Phi_{4}$ provides the best behavior than $\Phi_{2}$ while $\Phi_{1}$ is the poorest one. The corresponding quantitative measurement of errors is displayed in Figure 2. Error $E_{i}$ for each approximate solution $\Phi_{i}$ is defined by
Next, the approximate solutions $\Phi_{1}, \Phi_{2}, \Phi_{4}$ and the exact solution [26]

$$
E_{i}=\left|\frac{\Phi_{i}-U_{\text {exact }}}{U_{\text {exact }}}\right|, i=1,2,4
$$

In this figure $E_{1}, E_{2}$ and $E_{4}$ are displayed in green, blue and red curves, respectively. It is found that $E_{1}$ is around 0.1 while $E_{2}$ is smaller than 0.01 for most time domain. $E_{4}$ is much smaller than $E_{2}$ and $E_{1}$ which indicates again the rapid convergence of the present ADM solution.

Table 1. Values of parameters $a$ and $b$.

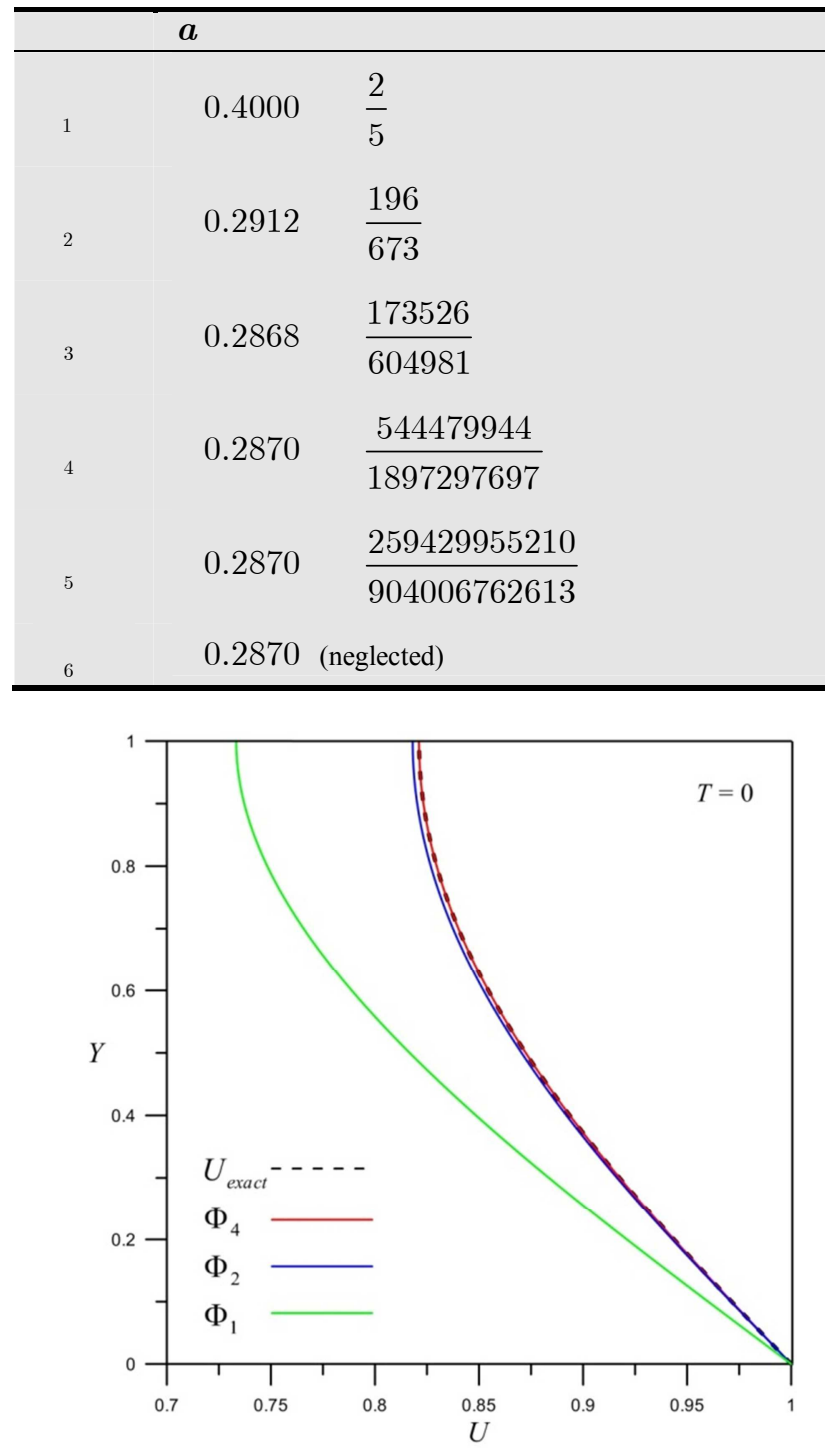

(a)
$0.8000 \quad \frac{4}{5}$
$0.8915 \quad \frac{600}{673}$
$0.8855 \quad \frac{535740}{604981}$
$0.8854 \quad \frac{1679959792}{1897297697}$
$0.8855 \quad \frac{800453511540}{904006762613}$
0.8855 (neglected)

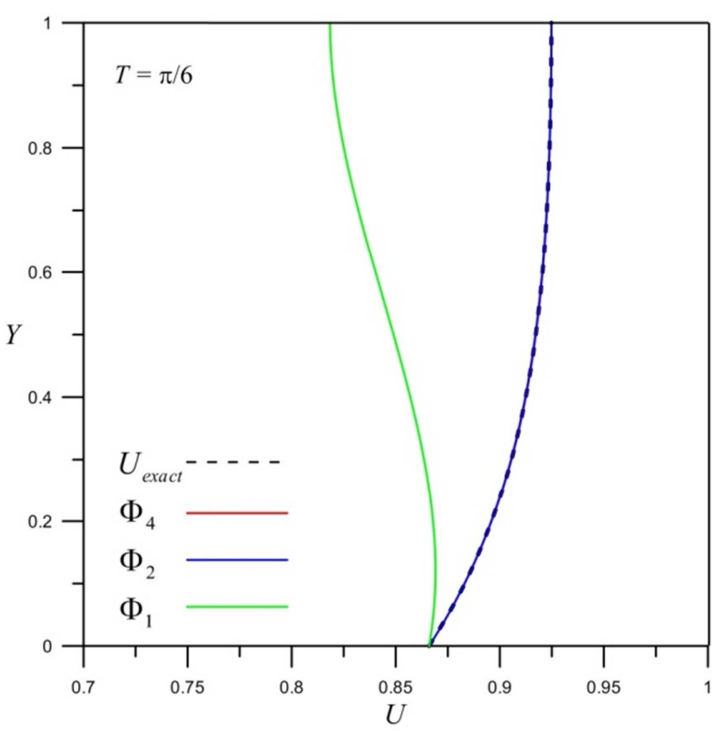

(b) 


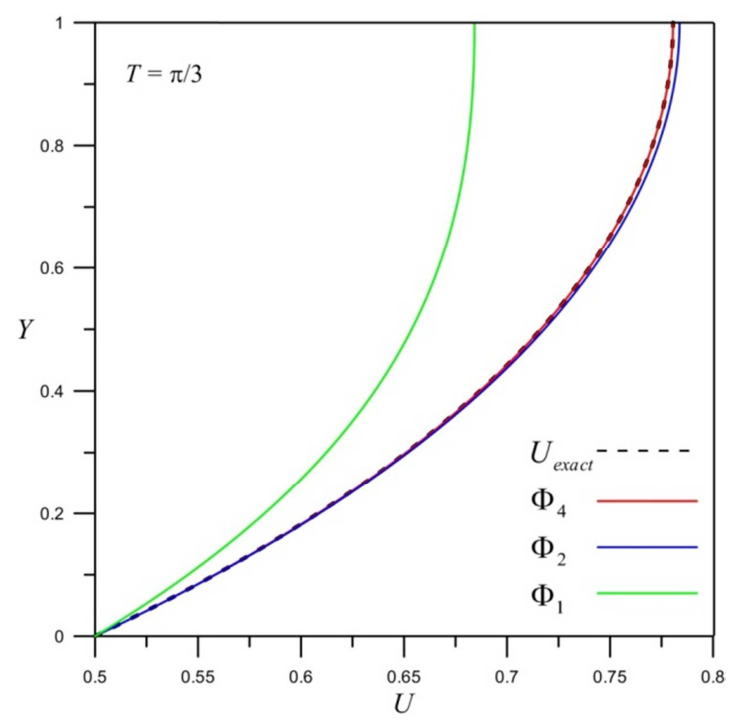

(c)

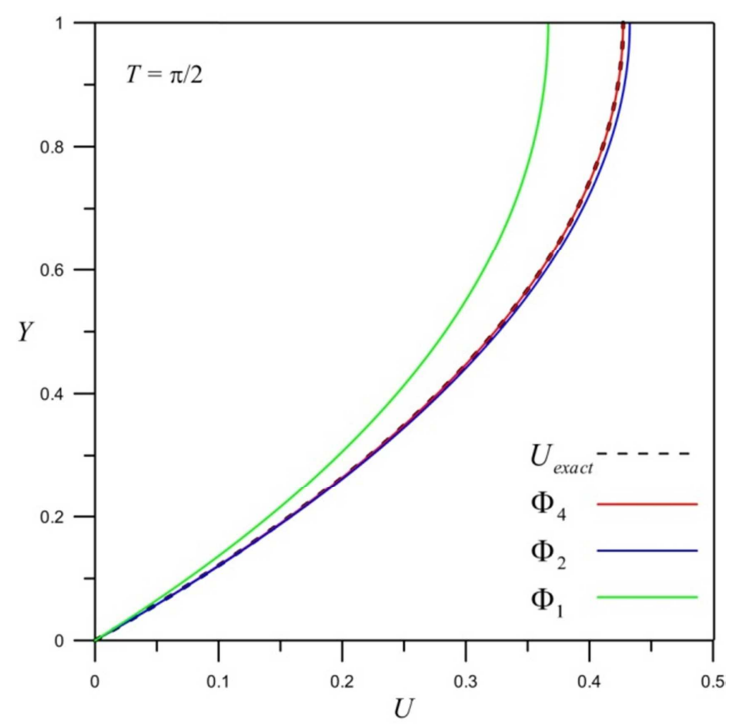

(d)

Figure 1. Velocity profiles for Stokes' second problem: (a) $T=0$, (b) $T=\pi / 6$, (c) $T=\pi / 3$ and (d) $T=\pi / 2$.

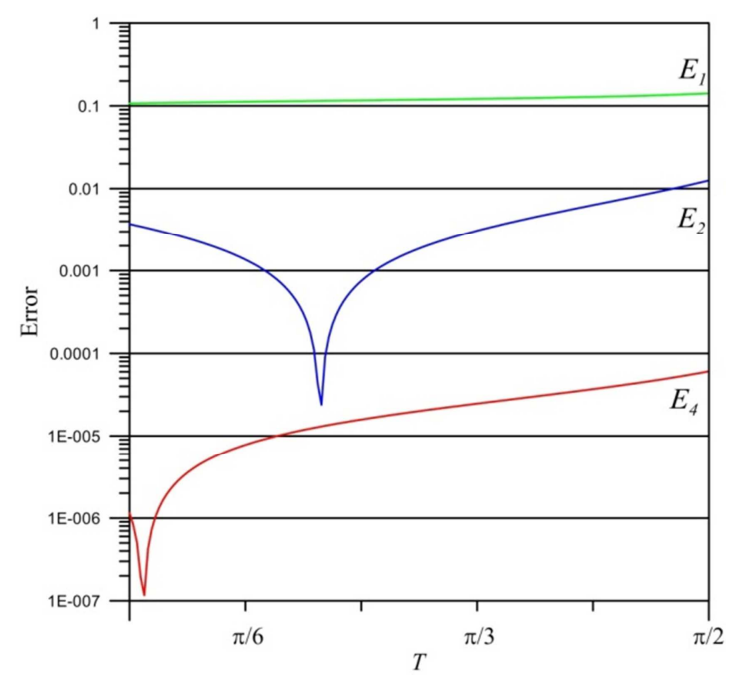

Figure 2. Errors of approximate solutions for Stokes'second problems.

\section{Pulsatile Flow Between Horizontal Plates}

A flow with periodic variation is known as pulsatile flow. It is commonly observed in arterial blood circulation, hydraulic machines and motor engines. Exact solutions of pulsatile flows between plates and in a circular tube are well known and are referred to [30] and [31]. In this section, a pressure-driven pulsatile flow bounded by two fixed horizontal plates is studied by ADM. An oscillating pressure gradient is the only driven force for the flow system. Liu [17] studied the same problem by assigning the velocity and its derivative at the middle plane between two plates. For the sake of simulating a real flow, the pressure gradient will be the only assignment in present study.

Consider a fluid of density $\rho$ and kinematic viscosity $v$ bounded by two plates at $y= \pm h$. The momentum equation is

$$
\frac{\partial u}{\partial t}=-\frac{1}{\rho} \frac{\partial p}{\partial x}+v \frac{\partial^{2} u}{\partial y^{2}},
$$

where the pressure gradient is given by

$$
\frac{\partial p}{\partial x}=p_{0} \cos t \omega
$$

As the flow velocity at plates is zero, the corresponding boundary conditions are

$$
u(y= \pm h)=0
$$

Applying the following dimensionless variables

$$
U=\frac{u}{h \omega}, Y=\frac{y}{h}, T=\omega t,
$$

to Eqs.(26) and (28), the results are

$$
\frac{\partial U}{\partial T}=-\eta \cos T+\varsigma \frac{\partial^{2} U}{\partial Y^{2}} .
$$

where

$$
\left\{\begin{array}{l}
\eta=\frac{p_{0}}{\rho \omega^{2} h} \\
\varsigma=\frac{v}{h^{2} \omega}
\end{array} .\right.
$$

In the following analysis $\eta=\varsigma=1$ will be assigned without loss of generality. The boundary conditions at both plates become

$$
U(Y= \pm 1)=0
$$

Now applying the operator defined in Eq.(8) to Eq.(30) leads to 


$$
\begin{array}{r}
\mathrm{L}_{Y} U=\cos T+\frac{\partial U}{\partial T} . \quad \text { (33) } \begin{array}{c}
\text { Applying the inverse operator of Eq.(10) } \\
\text { results in }
\end{array} \\
U(Y, T)=U(0, T)+Y \cdot U_{Y}(0, T)+\frac{Y^{2}}{2 !} \cdot \cos T+\int_{0}^{Y} \int_{0}^{Y} \frac{\partial U}{\partial T} \mathrm{~d} Y \mathrm{~d} Y .
\end{array}
$$

Applying the inverse operator of Eq.(10) to above equation

As the flow is symmetrical to $Y=0$, it implies that $U_{Y}(Y=0)$ has to be zero. The velocity at $Y=0$ is assumed to be $G(T)$ which will be determined later. Now the starting term of the decomposition series is given by

$$
U_{0}(Y, T)=G(T)+\frac{Y^{2}}{2 !} \cos T
$$

The remaining term can be recursively calculated by Eq.(14). It gives

$$
\left\{\begin{array}{l}
U_{1}=\frac{Y^{2}}{2 !} G^{\prime}(T)-\frac{Y^{4}}{4 !} \sin T \\
U_{2}=\frac{Y^{2}}{4 !} G^{\prime \prime}(T)-\frac{Y^{6}}{6 !} \cos T \\
U_{3}=\frac{Y^{6}}{6 !} G^{\prime \prime \prime}(T)+\frac{Y^{8}}{8 !} \sin T \\
U_{4}=\frac{Y^{8}}{8 !} G^{(4)}(T)+\frac{Y^{10}}{10 !} \cos T
\end{array} .\right.
$$

The complete solution is shown as

$$
U(Y, T)=\sum_{i=0}^{\infty} \frac{Y^{2 i} G^{(i)}(T)}{(2 i) !}+\cos T \cdot \sum_{i=0}^{\infty} \frac{(-1)^{i} Y^{4 i+2}}{(4 i+2) !}+\sin T \cdot \sum_{i=0}^{\infty} \frac{(-1)^{i+1} Y^{4 i+4}}{(4 i+4) !}
$$

Now the boundary condition at the upper plate, $U(Y=1)=0$, is adopted to calculate $G(T)$. The result is

$$
\sum_{i=0}^{\infty} \frac{G^{(i)}(T)}{(2 i) !}+\cos T \cdot \sum_{i=0}^{\infty} \frac{(-1)^{i}}{(4 i+2) !}+\sin T \cdot \sum_{i=0}^{\infty} \frac{(-1)^{i+1}}{(4 i+4) !}=0
$$

As the pressure gradient is oscillatory and so as the flow, $G(T)$ is assumed to be of the form

$$
G(T)=c \cos T+d \sin T,
$$

where $c$ and $d$ are unknowns to be determined. Substituting Eq.(39) into Eq.(38) gives

$$
\begin{aligned}
& \left\{c \cdot \sum_{i=0}^{\infty} \frac{(-1)^{i}}{(4 i) !}+d \cdot \sum_{i=0}^{\infty} \frac{(-1)^{i}}{(4 i+2) !}+\sum_{i=0}^{\infty} \frac{(-1)^{i}}{(4 i+2) !}\right\} \cdot \cos T \\
& +\left\{c \cdot \sum_{i=0}^{\infty} \frac{(-1)^{i+1}}{(4 i+2) !}+d \cdot \sum_{i=0}^{\infty} \frac{(-1)^{i}}{(4 i) !}+\sum_{i=0}^{\infty} \frac{(-1)^{i+1}}{(4 i+4) !}\right\} \cdot \sin T=0
\end{aligned}
$$

For the approximate solution $\Phi_{n}$, parameters $c$ and $d$ can solved by

$$
\left\{\begin{array}{l}
c \cdot \sum_{i=0}^{i \leq \frac{n}{2}} \frac{(-1)^{i}}{(4 i) !}+d \cdot \sum_{i=0}^{i \leq \frac{n}{2}-\frac{1}{2}} \frac{(-1)^{i}}{(4 i+2) !}=\sum_{i=0}^{i \leq \frac{n}{2}} \frac{(-1)^{i+1}}{(4 i+2) !} \\
c \cdot \sum_{i=0}^{i \leq \frac{n}{2}-\frac{1}{2}} \frac{(-1)^{i+1}}{(4 i+2) !}+d \cdot \sum_{i=0}^{i \leq \frac{n}{2}} \frac{(-1)^{i}}{(4 i) !}=\sum_{i=0}^{i \leq \frac{n}{2}-\frac{1}{2}} \frac{(-1)^{i}}{(4 i+4) !}
\end{array} .\right.
$$

Solution pairs of $c$ and $d$ for the first four approximate solutions are shown in Table 2. It is also found that $c$ and $d$ rapidly converge when the order of $\Phi$ grows. Figure 3 shows a comparison of $\Phi_{1}, \Phi_{2}, \Phi_{4}$ and the exact solution [30] 


$$
U_{\text {exact }}=\operatorname{Re}\left\{i e^{i T}\left[1-\frac{\cosh \left(\frac{(1+i) Y}{\sqrt{2}}\right)}{\cosh \left(\frac{(1+i)}{\sqrt{2}}\right)}\right]\right\},-1 \leq Y \leq 1 .
$$

As the parameters $c$ and $d$ converge very quickly, very slight difference exists between $\Phi_{2}, \Phi_{4}$ and the exact solution. This is also observed in Figure 4 which displays the quantitative measurement of errors defined by Eq.(25).

Table 2. Values of parameters $c$ and $d$.

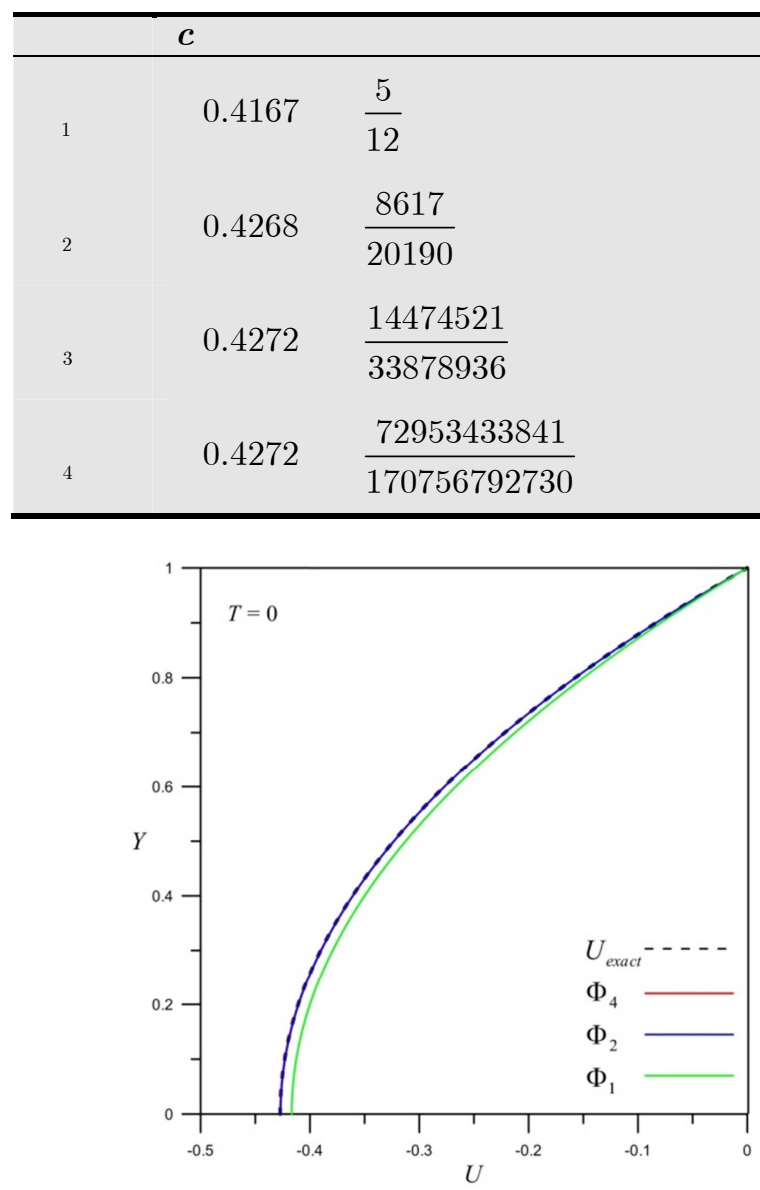

(a)

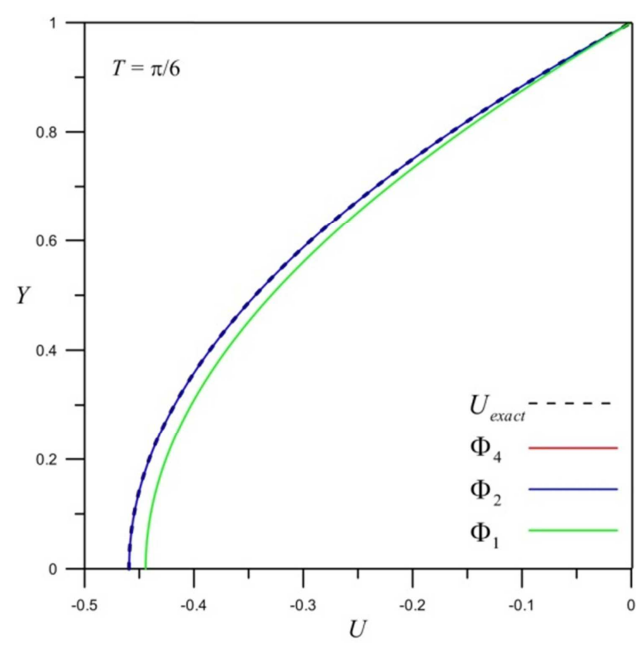

(b)
$0.1667 \frac{1}{6}$
$0.1792 \quad \frac{603}{3365}$
$0.1788 \quad \frac{3029413}{16939468}$
$0.1788 \quad \frac{15268176017}{85378396365}$

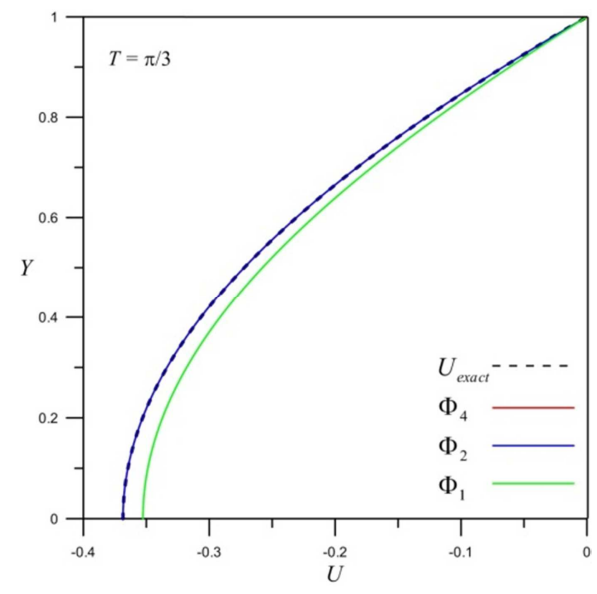

(c)

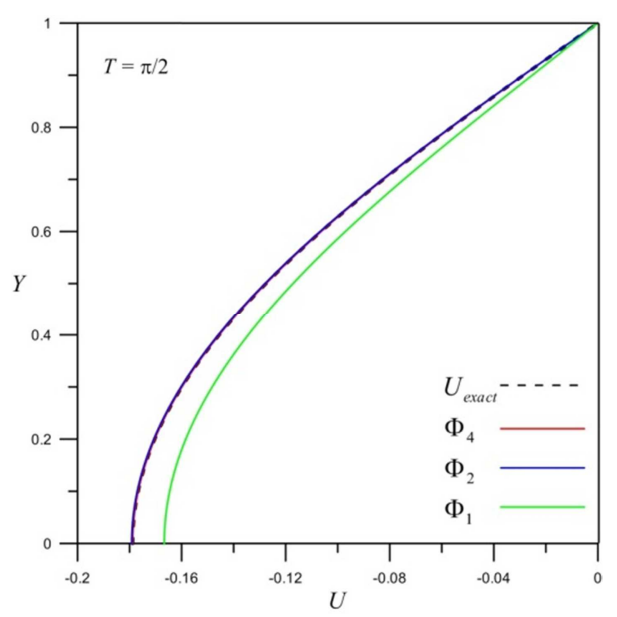

(d)

Figure 3. Velocity profiles for planar pulsatile flow: (a) $T=0$, (b) $T=\pi / 6$, (c) $T=\pi / 3$ and (d) $T=\pi / 2$. 


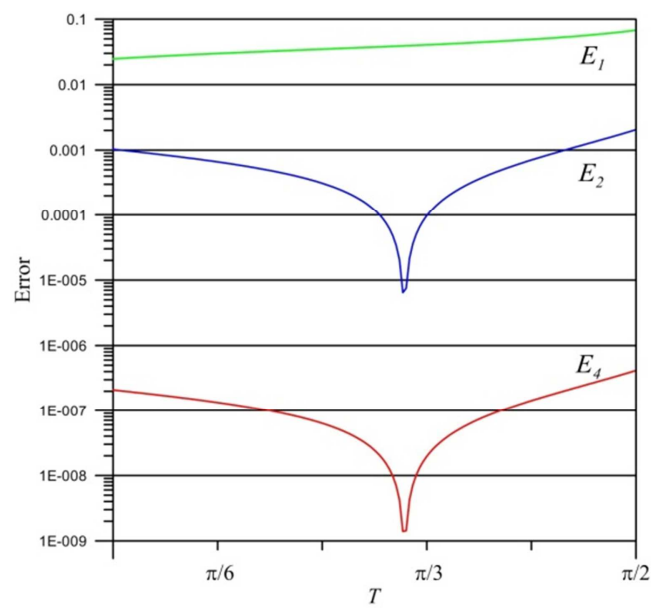

Figure 4. Errors of approximate solutions for planar pulsatile flow.

\section{Pulsatile Flow in a Circular Tube}

The problem studied in this section is almost the same as that in previous section, except the geometry is changed from a planar type to a circular type. Consider a viscous flow in a circular tube of radius $a$ driven by a pressure gradient, the momentum equation reads

$$
\frac{\partial u}{\partial t}=-\frac{1}{\rho} \frac{\partial p}{\partial x}+v\left(\frac{\partial^{2} u}{\partial r^{2}}+\frac{1}{r} \frac{\partial u}{\partial r}\right)
$$

where the pressure gradient is given by Eq.(27). As the flow velocity around the tube is zero due to viscosity, the corresponding boundary condition is

$$
u(r=a)=0 \text {. }
$$

Besides, according to the symmetrical geometry of the tube, it implies another boundary condition

$$
\frac{\partial u}{\partial r}(r=0)=0
$$

Applying the following dimensionless variables

$$
U(R, T)=U(0, T)+R \cdot U_{R}(0, T)+\mathrm{L}_{R}^{-1}\left[\frac{\partial U}{\partial T}+\cos T\right]
$$

which can be further written as

$$
U(R, T)=H(T)+\frac{1}{4} R^{2} \cos T+\mathrm{L}_{R}^{-1}\left[\frac{\partial U}{\partial T}\right]
$$

where $H(T)$ indicates the velocity at $Y=0$. Now the first term of the decomposition series is assigned by

$$
U_{0}(Y, T)=H(T)+\frac{R^{2}}{2^{2}} \cos T
$$

The remaining terms can be recursively calculated and are shown below

$$
U=\frac{u}{a \omega}, R=\frac{r}{a}, T=\omega t
$$

where

$$
\left\{\begin{array}{l}
\eta=\frac{p_{0}}{\rho \omega^{2} a} \\
\varsigma=\frac{v}{\omega a^{2}}
\end{array} .\right.
$$

Applying the inverse operator to above equation results in

In the following analysis $\eta=\varsigma=1$ will be assigned without loss of generality. Now we define the differential

$$
\mathrm{L}_{R}(*)=\frac{1}{R} \frac{\partial}{\partial R}\left(R \frac{\partial}{\partial R}(*)\right)
$$

and its inversion

$$
\mathrm{L}_{R}^{-1}(*)=\int_{0}^{R} R^{-1} \int_{0}^{R} R \cdot(*) d R d R
$$

and then apply the operator to Eq.(50), it gvies

$$
\mathrm{L}_{R} U=\cos T+\frac{\partial U}{\partial T} \text {. }
$$

.




$$
\left\{\begin{array}{l}
U_{1}=\frac{R^{2}}{2^{2}} H^{\prime}(T)-\frac{R^{4}}{(2 \cdot 4)^{2}} \sin T \\
U_{2}=\frac{R^{4}}{(2 \cdot 4)^{2}} H^{\prime \prime}(T)-\frac{R^{6}}{(2 \cdot 4 \cdot 6)^{2}} \cos T \\
U_{3}=\frac{R^{6}}{(2 \cdot 4 \cdot 6)^{2}} H^{\prime \prime \prime}(T)+\frac{R^{8}}{(2 \cdot 4 \cdot 6 \cdot 8)^{2}} \sin T \\
U_{4}=\frac{R^{8}}{(2 \cdot 4 \cdot 6 \cdot 8)^{2}} H^{(4)}(T)+\frac{R^{10}}{(2 \cdot 4 \cdot 6 \cdot 8 \cdot 10)^{2}} \cos T
\end{array}\right.
$$

The complete solution is displayed

$$
U(R, T)=\sum_{i=0}^{\infty} \frac{R^{2 i} H^{(i)}(T)}{\Psi(i)}+\sum_{i=0}^{\infty} \frac{R^{2 i+2} \cos T}{\Psi(i+1)} .
$$

Using the boundary condition $U(R=1)=0$ gives

$$
\sum_{i=0}^{\infty} \frac{H^{(i)}(T)}{\Psi(i)}+\sum_{i=0}^{\infty} \frac{\cos T}{\Psi(i+1)}=0
$$

where

$$
\Psi(i)= \begin{cases}1 & \text { for } i=0 \\ {[2 \cdot 4 \cdot \ldots \cdot(2 i)]^{2}} & \text { for } i \neq 0\end{cases}
$$

Similar to the idea adopted in previous section, $H(T)$ is assumed to be

$$
H(T)=e \cos T+f \sin T,
$$

where $e$ and $f$ are unknowns to be determined. Substituting Eq.(60) into Eq.(58) results in

$$
\begin{aligned}
& \left\{e \cdot \sum_{i=0}^{\infty} \frac{(-1)^{i}}{\Psi(2 i)}+f \cdot \sum_{i=0}^{\infty} \frac{(-1)^{i}}{\Psi(2 i+1)}+\sum_{i=0}^{\infty} \frac{(-1)^{i}}{\Psi(2 i+1)}\right\} \cdot \cos T \\
+ & \left\{e \cdot \sum_{i=0}^{\infty} \frac{(-1)^{i+1}}{\Psi(2 i+1)}+f \cdot \sum_{i=0}^{\infty} \frac{(-1)^{i}}{\Psi(2 i)}+\sum_{i=0}^{\infty} \frac{(-1)^{i+1}}{\Psi(2 i+2)}\right\} \cdot \sin T=0
\end{aligned}
$$

Now $e$ and $f$ for $\Phi_{n}$ can be determined by

$$
\left\{\begin{array}{l}
e \cdot \sum_{i=0}^{i \leq \frac{n}{2}} \frac{(-1)^{i}}{\Psi(2 i)}+f \cdot \sum_{i=0}^{i \leq \frac{n}{2}-\frac{1}{2}} \frac{(-1)^{i}}{\Psi(2 i+1)}=\sum_{i=0}^{i \leq \frac{n}{2}} \frac{(-1)^{i+1}}{\Psi(2 i+1)} \\
e \cdot \sum_{i=0}^{i \leq \frac{n}{2}-\frac{1}{2}} \frac{(-1)^{i+1}}{\Psi(2 i+1)}+f \cdot \sum_{i=0}^{i \leq \frac{n}{2}} \frac{(-1)^{i}}{\Psi(2 i)}=\sum_{i=0}^{i \leq \frac{n}{2}-\frac{1}{2}} \frac{(-1)^{i}}{\Psi(2 i+2)}
\end{array} .\right.
$$

Similar to the analysis in previous section, the convergence behavior of $e$ and $f$ shown in Table 3 is excellent. Figure 5 displays the velocity profiles along $R$ at four different times. Both $\Phi_{4}$ and $\Phi_{2}$ are almost the same as the exact solution while $\Phi_{1}$ behaves slightly poor. 
Table 3. Values of parameters $e$ and $f$.

\begin{tabular}{lllll}
\hline \multicolumn{2}{c}{$\boldsymbol{e}$} & & $f$ & \\
\hline 1 & 0.2390 & $\frac{65}{272}$ & 0.0441 & $\frac{3}{68}$ \\
2 & 0.2420 & $\frac{4089}{16900}$ & 0.0456 & $\frac{1733}{38025}$ \\
3 & 0.2420 & $\frac{84786625}{350364736}$ & 0.0455 & $\frac{3984199}{87591184}$ \\
4 & 0.2420 & $\frac{23592966311}{97494058300}$ & 0.0455 & $\frac{44345783}{974940583}$ \\
\hline
\end{tabular}

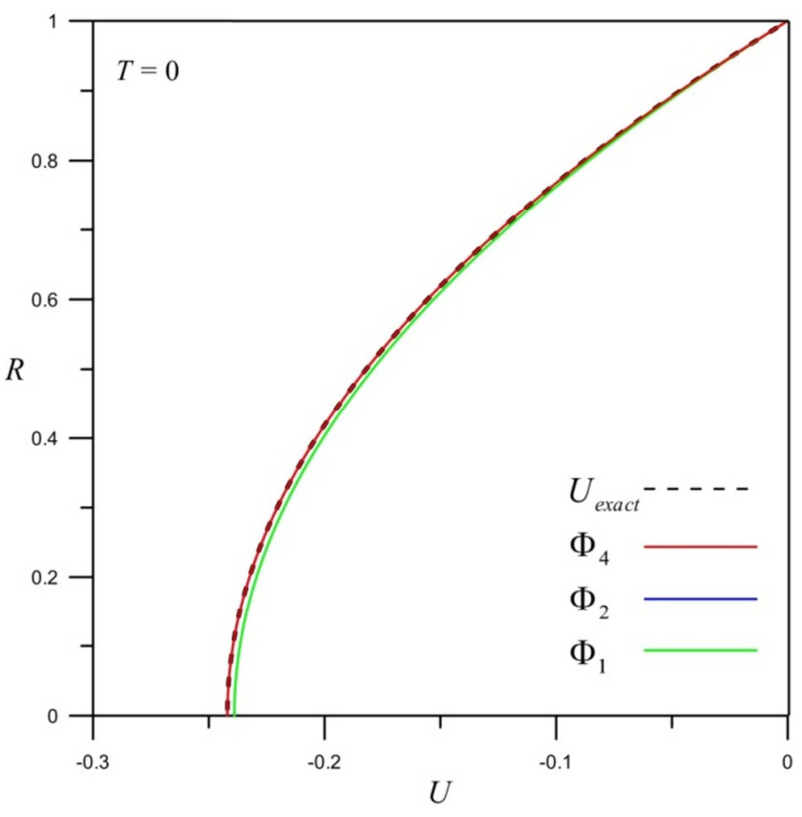

(a)

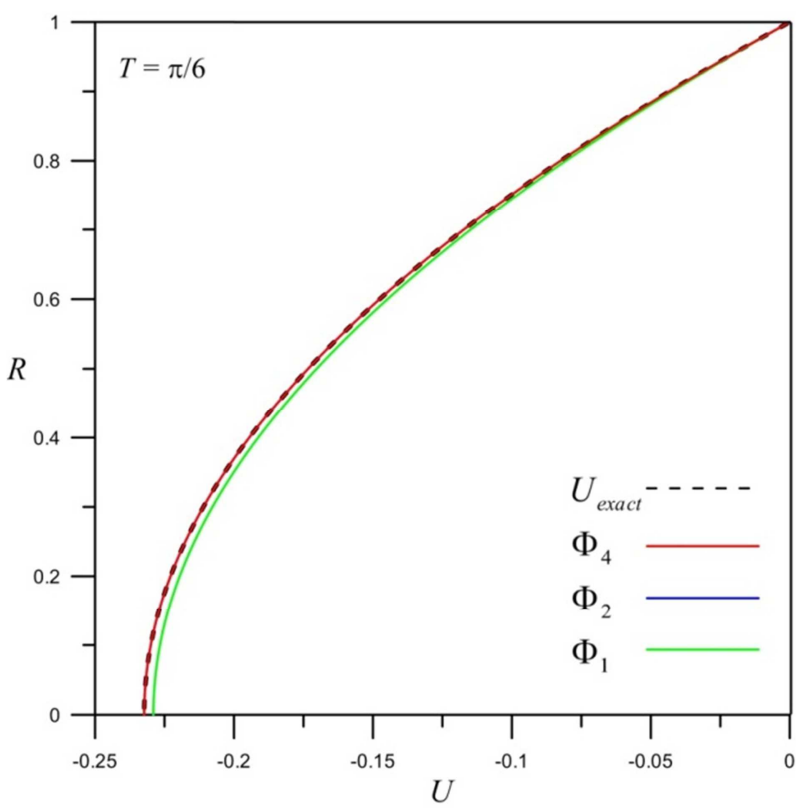

(b)

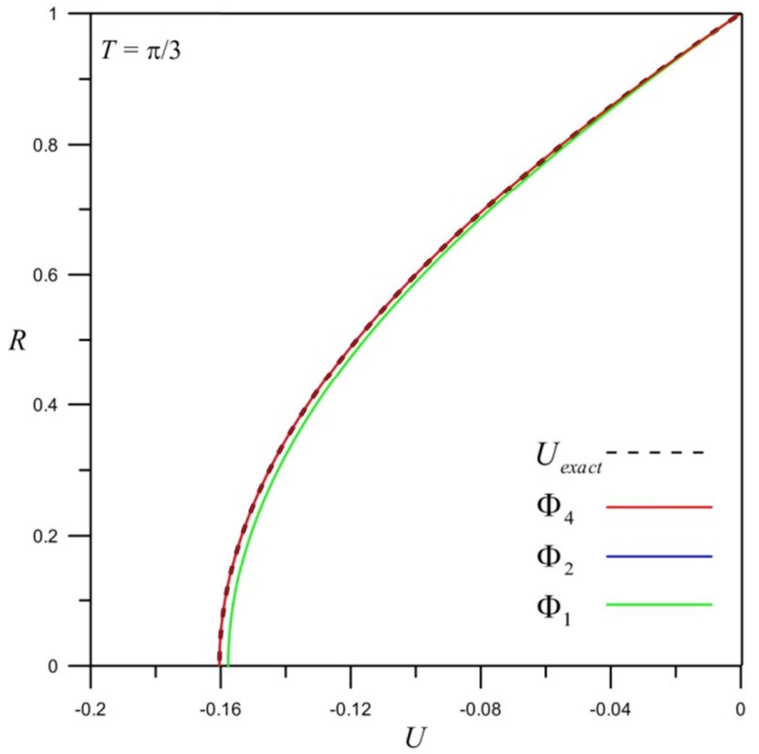

(c)

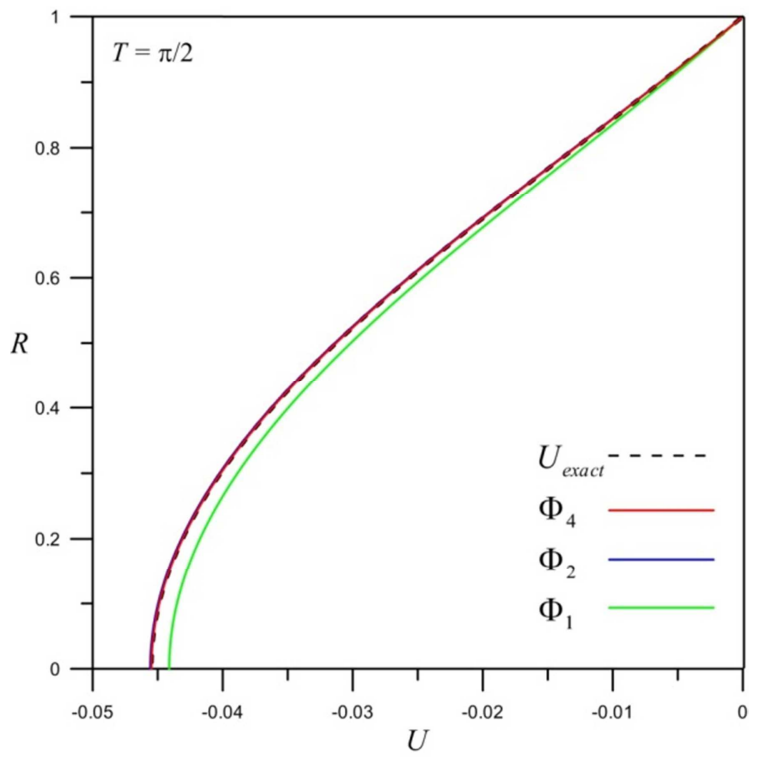

(d)

Figure 5. Velocity profiles for pulsatile flow in a circular tube: (a) $T=0$, (b) $T=\pi / 6,(c) T=\pi / 3$ and $(d) T=\pi / 2$. 


\section{Concluding Remarks}

In this paper three types of oscillating viscous flows are examined by applying Adomian decomposition method. Different from previous studies for the same problem, the assignment of the first term of the decomposition series is simply the plate velocity for Stokes' second problem and the pressure gradient for pulsatile flows. In each case approximate solution accurate up to any order can be easily acquired after two constant parameters are determined. It is found that two parameters and the corresponding approximate solution converge very rapidly for all cases. Present results show an excellent agreement with the exact solution which demonstrates the capacity of ADM for simulating realistic flows. Based on the present method, more complicated initial-value and boundary-value problems with periodic variations can be simulated or solved by ADM associated with the Fourier analysis in the future.

\section{Acknowledgements}

The financial support from Ministry of Science and Technology of Taiwan with the grants MOST-104-2221-E-270-003-MY2 and NSC-102-2911-I-006-302 is acknowledged.

\section{References}

[1] G. A. Adomian, "A review of the decomposition method in applied mathematics," Journal of Mathematical Analysis and Applications, Vol. 135, No. 2, pp. 501-544, 1988.

[2] G. A. Adomian, Solving frontier problems of physics: the decomposition method, Dordrecht, Kluwer Academic Pub., 1994.

[3] A. M. Wazwaz, "The numerical solution of fifth-order boundary value problems by the decomposition method," Journal of Computational and Applied Mathematics, Vol. 136, pp. 259-270, 2001.

[4] A. M. Wazwaz and S. M. El-Sayed, "A new modification of the Adomian decomposition method for linear and nonlinear operators," Applied Mathematics and Computation, Vol. 122, pp. 393-405, 2001.

[5] M. Dehghan, "Application of the Adomian decomposition method for two-dimensional parabolic equation subject to nonstandard boundary specifications," Applied Mathematics and Computation, Vol. 157, pp. 549-560, 2004.

[6] M. Dehghan, "The use of Adomian decomposition method for solving the one-dimensional parabolic equation with non-local boundary specifications," International Journal of Computer Mathematics, Vol. 81, pp. 25-34, 2004.

[7] M. M. Hosseini, "Adomian decomposition method for solution of differential-algebraic equations," Journal of Computational and Applied Mathematics, Vol. 197, pp. 495-501, 2006.

[8] I. Hashim, "Adomian decomposition method for solving BVPs for fourth-order integro-differential equations," Journal of Computational and Applied Mathematics, Vol. 193, pp. 658664, 2006.
[9] A. M. Wazwaz, "A comparison between the variational iteration method and Adomian decomposition method," Journal of Computational and Applied Mathematics, Vol. 207, pp. 129-136, 2007.

[10] A. H. Bokhari, G. Mohammad, M. T. Mustafa and F. D. Zaman, "Adomian decomposition method for a nonlinear heat equation with temperature dependent thermal properties," Mathematical Problems in Engineering, Vol. 2009, 926086, 2009.

[11] J. S. Duan, "An efficient algorithm for the multivariable Adomian polynomials," Applied Mathematics and Computation, Vol. 217, pp. 2456-2467, 2010.

[12] A. Wazwaz, "The modified decomposition method and Padé approximation for solving the Thomas-Fermi equation," Applied Mathematics and Computation, Vol. 102, pp. 11-19, 1999.

[13] M. Dehghan, A. Hamidi and M. Shakourifar, "The solution of coupled Burgers' equations using Adomian-Padé technique," Applied Mathematics and Computation, Vol. 189, pp. 1034-1047, 2007.

[14] T. A. Abassy, M. A. El-Tawil and H. K. Saleh, "The solution of Burgers' and good Boussinesq equations using ADM-Padé technique," Chaos, Solitons and Fractals, Vol. 32, pp. 1008-1026, 2007.

[15] Y. Cherruault and G. A. Adomian, "Decomposition method: a new proof of convergence," Mathematical and Computer Modelling, Vol. 18, pp. 103-106, 1993.

[16] D. Lesnic, "Convergence of Adomian' decomposition method: periodic temperatures," Computers \& Mathematics with Applications, Vol. 44, pp. 13-24, 2002.

[17] C. M. Liu, "On the study of oscillating viscous flows by using the Adomian-Padé approximation," Journal of Applied Mathematics, Vol. 2015, 864190, 2015.

[18] M. E. Erdogan, "A note on an unsteady flow of a viscous fluid due to an oscillating plane wall," International Journal of Non-Linear Mechanics, Vol. 35, pp. 1-6, 2000.

[19] C. M. Liu and I. C. Liu, "A note on the transient solution of Stokes' second problem with arbitrary initial phase," Journal of Mechanics, Vol. 22, No. 4, pp. 349-354, 2006.

[20] F. Farkhadnia, R. Kamrani and D. D. Ganji, "Analytical investigation for fluid behavior over a float plate with oscillating motion and wall transpiration," New Trends in Mathematical Sciences, Vol. 2, pp. 178-189, 2014.

[21] C. M. Liu, "Extended Stokes' problems of relatively moving porous half-planes," Mathematical Problems in Engineering, Vol. 2009, 185965, 2009.

[22] D. O. de Almeida Cruz and E. F. Lins, "The unsteady flow generated by an oscillating wall with transpiration," International Journal of Non-linear Mechanics, Vol. 45, pp. 453-457, 2010.

[23] G. G. Stokes, "On the effect of the internal friction of fluids on the motion of pendulums," Transactions of the Cambridge Philosophical Society, Vol. 9, pp. 8-106, 1851.

[24] L. Rayleigh, "On the motion of solid bodies through viscous liquid," Philosophical Magazine, Vol. 6, pp. 697-711, 1911.

[25] R. Panton, "The transient for Stokes' oscillating plate: a solution in terms of tabulated functions," Journal of Fluid Mechanics, Vol. 31, pp. 819-825, 1968. 
[26] C. M. Liu, "Complete solutions to extended Stokes' problems," Mathematical Problems in Engineering, Vol. 2008, 754262, 2008.

[27] L. Ai and K. Vafai, "An investigation of Stokes' second problem for non-Newtonian fluids," Numerical Heat Transfer, Part A, Vol. 47, pp. 955-980, 2005.

[28] D. Pritchard, C. R. McArdle and S. K. Wilson, "The Stokes boundary layer for a power-law fluid," Journal of Non-Newtonian Fluid Mechanics, Vol. 166, pp. 745-753, 2011.
[29] S. P. Hu, C. M. Fan, C. W. Chen and D. L. Young, "Method of fundamental solutions for Stokes' first and second problems," Journal of Mechanics, Vol. 21, pp. 25-13, 2005.

[30] I. G. Currie, Fundamental Mechanics of Fluids, $2^{\text {nd }}$ ed., McGraw-Hill, New York, 1993.

[31] Y. C. Fung, Biomechanics: motion, flow, stress, and growth, Springer, New York, 1990. 